\title{
A General Mechanism Design Methodology for Social Utility Maximization with Linear Constraints
}

\author{
Abhinav Sinha and Achilleas Anastasopoulos \\ EECS Department, University of Michigan, Ann Arbor, MI 48109 \\ \{absi, anastas\}@umich.edu
}

September 11, 2018

\begin{abstract}
Social utility maximization refers to the process of allocating resources in such a way that the sum of agents' utilities is maximized under the system constraints. Such allocation arises in several problems in the general area of communications, including unicast (and multicast multi-rate) service on the Internet, as well as in applications with (local) public goods, such as power allocation in wireless networks, spectrum allocation, etc. Mechanisms that implement such allocations in Nash equilibrium have also been studied but either they do not possess full implementation property, or are given in a case-by-case fashion, thus obscuring fundamental understanding of these problems.

In this paper we propose a unified methodology for creating mechanisms that fully implement, in Nash equilibria, social utility maximizing functions arising in various contexts where the constraints are convex. The construction of the mechanism is done in a systematic way by considering the dual optimization problem. In addition to the required properties of efficiency and individual rationality that such mechanisms ought to satisfy, three additional design goals are the focus of this paper: a) the size of the message space scaling linearly with the number of agents (even if agents' types are entire valuation functions), b) allocation being feasible on and off equilibrium, and c) strong budget balance at equilibrium and also off equilibrium whenever demand is feasible.
\end{abstract}

Terms: Mechanism Design, Information Elicitation, Foundations of Incentive Compatibility, Auction Theory.

Key Words and Phrases: Multicast routing, proportional allocation, game theory, mechanism 


\section{Introduction}

In the general area of communications, a number of decentralized resource allocation problems have been studied in the context of mechanism design. Such problems include unicast service on the Internet [Yang and Hajek, 2005], [Maheswaran and Basar, 2004], [Kakhbod and Teneketzis, 2013a], multi-rate multicast service on the Internet [Kakhbod and Teneketzis, 2013b], [Sinha and Anastasopoulos, 2013], power allocation in wireless networks [Sharma and Teneketzis, 2012], [Han, 2012], [Zhang and Guizani, 2011], spectrum allocation [Huang et al., 2006] and pricing in a peer-to-peer network [Neely, 2009]. The mechanism design framework is both appropriate and useful in the above problems since these problems are motivated by the designer's desire to allocate resources efficiently in the presence of strategic agents who possess private information about their level of satisfaction from the allocation.

Usually mechanism design solutions define a contract such that the induced game has at least one equilibrium (Nash, Bayesian Nash, dominant strategy, etc) that corresponds to the desired allocation. This is usually obtained with direct mechanisms by appealing to the revelation principle [Börgers, 2013] [Garg et al., 2008a]. The drawbacks of these direct approaches is that they require agents to quote their types (which may be entire valuation functions) and that the induced game may have other extraneous equilibria that are not efficient. In this paper, the focus is on full Nash implementation. Without going into a formal definition, full Nash implementation refers to the design of contracts such that only the designer's most preferred outcome is realized as a result of interaction between strategic agents (i.e. at Nash equilibria ( $\mathrm{NE})$ ), as opposed to general mechanism design, where other less preferred outcomes are also possible. Thus implementation is more stringent and is capable of producing better allocations. Readers may refer to [Jackson, 2001] for a survey on implementation theory.

Concentrating on those proposed solutions in the literature that guarantee full Nash implementation, one observes a fragmented and case-by-case approach. One may ask how fundamentally different are for example the problems of unicast service on the Internet, multicast multi-rate service on the Internet, power allocation in wireless networks, etc, to justify a separately designed mechanism for each case. Alternatively, one may ask what are the common features in all these problems that can lead to a unified mechanism design approach. These questions provide the motivation for the work presented in this paper.

In particular, our starting point is to state a class of problems as social utility maxi-

design, full implementation, Nash equilibrium. 
mization under linear inequality/equality constraints. We then proceed by characterizing their solution through Karush-Kuhn-Tucker (KKT) conditions. Analyzing the dual optimization problem is essential in our approach, because it hints at how the taxation part of the mechanism should be designed. Subsequently we define a general mechanism and show that it results in full Nash implementation when the agents' valuation functions are their private information 3 . Since the application domain of interest is in the area of communications, we give special emphasis on the size of the message space (as a consequence, VCG-type mechanisms, see Krishna, 2009, Section 5.3], [Börgers, 2013, Chapter 5], [Shoham and Leyton-Brown, 2009, Chapter 10], [Garg et al., 2008a], [Garg et al., 2008b], are inappropriate since they require quoting of types, which are entire valuation function in this set-up). A notable exception is the work (Johari and Tsitsiklis, 2009, Section 5] and [Yang and Hajek, 2007] that adapts VCG for a small message space (one dimensional message per user) and guarantees off-equilibrium feasibility. However in their work, full implementation can't be guaranteed and there is possibility of extraneous equilibria. In this paper, the message space scales linearly with number of agents so that the proposed mechanism is scalable.

In addition to the stated goal of getting optimal allocations at all Nash equilibria, there are other auxiliary properties that are sought in a mechanism. An important such property is individual rationality; this requires that agents are weakly better off at $\mathrm{NE}$ than not participating in the mechanism at all. The purpose of this is to ensure that agents are willing to sign the contract in the first place. Another important property is strong budget balance ( $\mathrm{SBB}$ ) at $\mathrm{NE}$. This requires that at $\mathrm{NE}$, the total monetary transfer between agents (assuming quasi-linear utility functions) is zero. The unified mechanism proposed in this work possesses both these properties.

Finally, a mechanism may be endowed with auxiliary properties off equilibrium. These are meant to improve the applicability in practical settings. For instance, the NE is interpreted as the convergent outcome when agents in the system "learn" the game by playing it repeatedly, which implies that during this process the messages (and thus allocations and taxes) are off equilibrium. In our opinion, the most important auxiliary property is feasibility of allocation on and off equilibrium. This property is essential whenever the system constraints are hard constraints on resources and cannot be violated by any means. For instance, the contract should not promise rate allocations to agents that ever violate the capacity constraints of the network links because such a contract would be

\footnotetext{
${ }^{3}$ Use of Nash equilibrium as a solution concept itself requires some justification, since it applies only to games with complete information. This issue is discussed further in Section [5, However, in this work we accept the justification given by Reichelstein and Reiter in [Reichelstein and Reiter, 1988] and Groves and Ledyard in [Groves and Ledyard, 1977] based on the second interpretation of Nash equilibrium as the fixed point of an (possibly myopic) adjustment process, [Nash, 1950].
} 
practically invalid since it promises something that can never be achieved. Note that, at equilibrium the allocation has to satisfy the constraints by definition, so feasibility always holds at NE. Another auxiliary property is SBB off equilibrium. Similarly this property guarantees that in a practical situation where a dynamic learning process converges to $\mathrm{NE}$, each step of this process leaves a zero balance. One of the main features of the work in this paper is that the allocation scheme is designed to guarantee both feasibility off equilibrium and SBB off equilibrium whenever demand is feasible. Specifically, feasibility is achieved by utilizing proportional allocation. Proportional allocation refers to the idea of using agents' demands to create their allocation by projecting their overall demand vector back down to the boundary of the feasible region whenever it is outside of it. This way everyone receives allocation that is proportional to their original demand. This allocation method generalizes the idea of proportional allocation that was introduced in mechanism design framework by [Maheswaran and Basar, 2004], [Yang and Hajek, 2005] for unicast network with one capacity constraint and for stochastic control of networks in [Kelly et al., 1998], as well as by the authors in [Sinha and Anastasopoulos, 2014], [Sinha and Anastasopoulos, 2013].

The main contributions of this work are summarized as follows.

- A unified framework is proposed for full implementation (in NE) mechanism design in social utility maximization problems with convex constraints.

- The proposed mechanism has small message space (although agents' types are entire valuation functions) and is scalable.

- The proposed mechanism is individually rational and strongly budget balanced at NE.

- The proposed mechanism lends itself to practical implementation (through learning algorithms) because allocation is feasible even off equilibrium (and is strongly budget balanced off equilibrium when demand is feasible).

The rest of the paper is structured as follows. In Section 2 we describe three examples relevant in communications and formulate the general centralized allocation problem that we wish to implement. In Section 3 we describe the proposed mechanism. Section 4 contains all the proofs of full implementation. Section 5 contains off-equilibrium results and discusses their relevance. Finally in Section 6 we conclude with a discussion of important generalizations of this set-up. 


\section{Motivation and Centralized Problem}

In this section we start by describing various important resource allocation problems that arise in communications and to which our generalized methodology applies (in Section 6 we discuss generalizations that will help solve an even larger class of problems than the ones described below). After the examples, we define a general centralized optimization problem, (CP), that covers all the examples. Following that we state general assumptions on ( $(\mathrm{CP})$ (some additional technical assumptions will be made in Section 4).

\subsection{Interesting Resource allocation problems in Communications}

\section{Unicast Transmission on the Internet}

Consider agents on the Internet from set $\mathcal{N}=\{1, \ldots, N\}$, where each agent $i \in \mathcal{N}$ is a pair of source and destination that communicate via a pre-decided route consisting of links in $\mathcal{L}_{i}$. All the agents together communicate on the network consisting of links in $\mathcal{L}=\cup_{i \in \mathcal{N}} \mathcal{L}_{i}$. Since each link in the network has a limited capacity, this results in constraints on the information rate allocated to each agent. Considering a scenario where agents have (concave) utility functions/profiles $\left\{v_{\mathfrak{i}}(\cdot)\right\}_{\boldsymbol{i} \in \mathcal{N}}$ that measure the satisfaction received by agents for various allocated rates, we can write the social utility optimization problem as

$$
\begin{aligned}
& \max _{x \in \mathbb{R}_{+}^{N}} \sum_{i \in \mathcal{N}} v_{i}\left(x_{i}\right) \\
& \text { s.t. } \sum_{i \in \mathcal{N}^{l}} \alpha_{i}^{l} x_{i} \leqslant c^{l} \quad \forall l \in \mathcal{L} \text {. }
\end{aligned}
$$

In the above $\mathcal{N}^{l} \triangleq\left\{i \in \mathcal{N} \mid l \in \mathcal{L}_{i}\right\}$ is the set of agents present on link $l, \mathrm{c}^{l}$ is the capacity of link $l$ and $\alpha_{i}^{l}$ are positive weights meant to differentiate between true information rate $x_{i}$ and its imposition on capacity of the links of the network through coding rate, packet error etc.

The above feasible set is a polytope in the first quadrant of $\mathbb{R}_{+}^{N}$ and is created by faces that have outward normal vectors pointing away from the origin. For the details of a full implementation mechanism specifically for the unicast problem readers may refer to [Jain and Walrand, 2010], [Kakhbod and Teneketzis, 2013a], [Sinha and Anastasopoulos, 2014]. 


\section{Public Goods}

In contrast to the private consumption problem above, there are public goods problems where the resources are shared directly between agents instead of sharing via constraints. The unicast problem was a scenario where allocation of rate to one agent on a link would imply less available rate for other agents on the link (because of the capacity constraint) - the rate allocation thus is private in this case. In contrast to this, if there is a resource allocation problem where two or more agents can simultaneously use the same resource then it will be classified as a public goods problem. A well-studied example of this kind is the wireless transmission with interference, in which the power level of each agent affects any other agent through the signal-to-interference-and-noise ratio (SINR).

Here we consider a simplified scalar version of the public goods problem for a system of agents from the set $\mathcal{N}$ in the following form

$$
\begin{gathered}
\max _{x \in \mathbb{R}_{+}} \sum_{i \in \mathcal{N}} v_{i}(x) \\
\text { s.t. } \quad x \in X,
\end{gathered}
$$

where $X$ is a convex subset of $\mathbb{R}_{+}$. Note that the argument for all utility functions is the same; since there is one public good being simultaneously used by all the agents (for which $x$ marks the usage level). We rewrite such a problem in the constrained form

$$
\begin{gathered}
\max _{\underline{\underline{x} \in \mathbb{R}_{+}^{N}}} \sum_{i \in \mathcal{N}} v_{i}\left(x_{i}\right) \\
\text { s.t. } \quad x_{1}=x_{2}=x_{3}=\ldots=x_{N} \\
\text { s.t. } \quad x_{1} \in X .
\end{gathered}
$$

The treatment when $x$ is a vector is not very different and we discuss this in the generalizations at the end. Implementation for the public goods problem is the most studied of all the examples in this paper, see [Groves and Ledyard, 1977], [Hurwicz, 1979], [Chen, 2002]. The distinction from private goods is that generally public goods problems require handling the "free-rider" problem [Mas-Colell et al., 1995, Section 11.C].

\section{Local Public Goods}

Another important resource allocation problem in communications is the local public goods problem. The basic idea of direct sharing of resources is same as above but in here the sharing is only among agents locally. So there are local groups of agents for whom the allocation has to be the same, but this common allocation can be different from one 
group to the next. If we divide the set of agents into disjoint local groups: $\mathcal{N}=\sqcup_{k \in \mathcal{K}} \mathcal{N}_{k}$, then the centralized problem can be written as

$$
\begin{aligned}
& \max _{x \in \mathbb{R}_{+}^{k}} \sum_{k \in \mathcal{K}} \sum_{i \in \mathcal{N}_{k}} v_{i}\left(x_{k}\right) \\
& \text { s.t. } \quad x_{k} \in \mathcal{X}_{k} \quad \forall k \in \mathcal{K},
\end{aligned}
$$

with $\left(X_{k}\right)_{k \in \mathcal{K}}$ being convex subsets of $\mathbb{R}_{+}$. As before, we would restate it as

$$
\begin{gathered}
\max _{x \in \mathbb{R}_{+}^{k}} \sum_{k \in \mathcal{K}} \sum_{i \in \mathcal{N}_{k}} v_{i}\left(x_{i}\right) \\
\text { s.t. } \quad x_{i}=x_{j} \quad \forall i, j \in \mathcal{N}_{k}, \forall k \in \mathcal{K} \\
\text { s.t. } \quad x_{j_{k}} \in \mathcal{X}_{k} \text { for some } j_{k} \in \mathcal{N}_{k}, \forall k \in \mathcal{K} .
\end{gathered}
$$

Local public goods problems are relevant in those network settings where there is direct interaction between local agents. Wireless transmission is an example of this, each agent affects and is affected by other agents' transmission through interference (SINR) and it is reasonable to assume that this effect is only local and that agents situated far enough (either spatially or in the frequency domain) will not affect each other. Readers may refer to [Sharma and Teneketzis, 2012] for a specific mechanism for the local public goods problem.

\subsection{General Centralized Problem}

Here we state the generic form of the centralized optimization problem that we wish to fully implement. The resource allocation problem is defined for a system with agents indexed in the set $\mathcal{N}=\{1,2, \ldots, N\}$, who have utility functions $\left\{v_{i}(\cdot)\right\}_{i \in \mathcal{N}}$. The objective is to find the optimum allocation of a single infinitely divisible good that maximizes the sum of utilities subject to constraints on the system. The allocation made to agents will be denoted by the vector $x \in \mathbb{R}_{+}^{N}$, with $x_{i}$ being the allocation to agent $i$. The centralized optimization problem that we consider is

$$
\begin{gathered}
\max _{x} \sum_{i \in \mathcal{N}} v_{i}\left(x_{i}\right) \\
\text { s.t. } x \in \mathbb{R}_{+}^{N} \\
\text { s.t. } A_{l}^{\top} x \leqslant c_{l} \quad \forall l \in \mathcal{L} \text { where } A_{l} \in \mathbb{R}^{N}, c_{l} \geqslant 0 .
\end{gathered}
$$

The set $\mathcal{L}=\{1,2, \ldots, \mathrm{L}\}$ indexes all the constraints and $A_{l}, c_{l}$ are all parameters of the optimization problem. It is easy to see that the above set-up covers equality constraints 
(such as from the public goods example), since we can always write $x_{1}=\ldots=x_{N}$ as $x_{1} \geqslant x_{2} \geqslant \ldots \geqslant x_{N} \geqslant x_{1}$.

Denote by $\mathcal{C} \subset \mathbb{R}_{+}^{N}$ the above feasible set. Note that $\mathcal{C}$ is a polytope in the first quadrant of $\mathbb{R}^{\mathrm{N}}$, possibly of a lower dimension than $\mathrm{N}$ (due to equality constraints). For convenience, we denote by $\mathcal{L}_{i}$ the set of constraints that involve agent $i$, i.e. $\mathcal{L}_{i}=\{l \in$ $\left.\mathcal{L} \mid A_{l i} \neq 0\right\}$ with $L_{i} \triangleq\left|\mathcal{L}_{i}\right|$. Conversely, define $\mathcal{N}^{l}$ as the set of agents involved at link $l$ i.e. $\mathcal{N}^{l}=\left\{i \in \mathcal{N} \mid l \in \mathcal{L}_{i}\right\}$ with $N^{l} \triangleq\left|\mathcal{N}^{l}\right|$.

\subsection{Assumptions}

Stated below are the assumptions on (CP) some of which restrict the environment $\left\{v_{i}\right\}_{i \in \mathcal{N}}$ and some the constraint set $\mathcal{C}$. Some additional technical assumptions will be introduced later to handle the degenerate cases.

$$
\begin{aligned}
& \text { For any } i \in \mathcal{N}, v_{i}(\cdot) \text { is a strictly concave and continuously double differentiable } \\
& \text { function } \mathbb{R}_{+} \rightarrow \mathbb{R} \text {. }
\end{aligned}
$$

The purpose of strict concavity is to have a (CP) whose solution can be described sufficiently by the KKT conditions (note that monotonicity is not assumed).

The optimal solution $x^{\star}$ is bounded such that $x^{\star} \in \times_{i=1}^{N}\left(d_{i}, D\right)$ for some $0<$ $d_{i}<D$, with $d=\left(d_{i}\right)_{i=1}^{N} \in \mathcal{C}$ being arbitrarily close to $\underline{0}$ and $D$ being large enough.

This assumption is used to eliminate corner cases of ([CP), since they usually require special treatment and make the exposition unnecessarily convoluted. Note that we can always select a point $d \in \mathcal{C}$ that is arbitrarily close to $\underline{0}$ because of assumption (A3) below.

The next two assumptions restrict the constraint set $\mathrm{C}$.

(A3) The vector $\underline{0} \in \mathcal{C}$, i.e. $x=\underline{0}$ is feasible.

Since we are considering problems where all the variables $x_{i}$ have a physical interpretation, it is natural to consider a constraint set whereby every agent getting 0 allocation is feasible. Note that this also explains the choice $c_{l} \geqslant 0$.

For any constraint $l \in \mathcal{L}$ in $\left(C_{2}\right)$ there are two distinct $i, j \in \mathcal{N}$ such that $A_{l i}, A_{l j} \neq 0$. This can also be stated as $N^{l} \geqslant 2 \forall l \in \mathcal{L}$ where $N^{l}$ is as defined after ([CP). 
This ensures that there is indeed competition for all the constraints that could possibly be active at optimum. Again this is used to avoid special treatment of corner cases.

Denote by $\mathcal{V}_{0}$ the set of all possible functions $\left\{v_{i}(\cdot)\right\}_{i \in \mathcal{N}}$ that satisfy the above assumptions. Then $\nu_{0}$ will be the environment for our mechanism design problem.

We will also make an assumption on the overall utility of agents in the system.

Apart from the valuation part $v_{\mathfrak{i}}\left(x_{i}\right)$ there is linear taxation component as well
that affects agents' utilities. So overall utility of agent $i$ is
\[ u_{i}(x, t)=v_{i}\left(x_{i}\right)-t_{i} \]

\section{$2.4 \mathrm{KKT}$ conditions}

The Lagrangian for the optimization problem (ICP) is

$$
\mathrm{L}(x, \lambda, \mu)=\sum_{i \in \mathcal{N}} \nu_{i}\left(x_{i}\right)-\sum_{l \in \mathcal{L}} \lambda_{l}\left(A_{l}^{\top} x-c_{l}\right)+\sum_{i \in \mathcal{N}} \mu_{i} x_{i}
$$

Due to assumption (A2), we can state the KKT conditions only in terms of $\lambda^{\star}$ and not involve $\mu^{\star}$.

1. Primal Feasibility: $\chi^{\star} \in \mathcal{C}$.

2. Dual Feasibility: For all $l \in \mathcal{L}, \lambda_{l}^{\star} \geqslant 0$.

3. Complimentary Slackness: For all $l \in \mathcal{L}$,

$$
\lambda_{l}^{\star}\left(A_{l}^{\top} x^{\star}-c_{l}\right)=0 .
$$

4. Stationarity: For all $i \in \mathcal{N}$,

$$
v_{i}^{\prime}\left(x_{i}^{\star}\right)=\sum_{l \in \mathcal{L}} A_{l i} \lambda_{l}^{\star} .
$$

We will see later that taxation will help us in achieving these KKT conditions when agents play the induced game from the mechanism. For that it will be important to think of the Lagrange multipliers above as "prices" where there will be one price per constraint. 


\section{Mechanism}

In this section we describe the proposed mechanism. Description of the mechanism is divided into two parts - allocation and taxes. A mechanism in the Hurwicz-Reiter framework consists of an environment, an outcome space, a (centralized) correspondence between the environment and the outcome space, a message space and a contract from the message space to the outcome space. In our case the environment is set $\nu_{0}$ of all

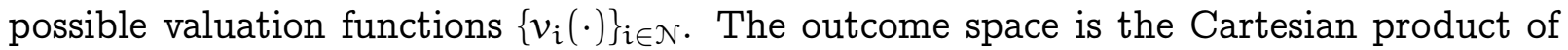
the set of all possible allocations and taxes, which is the set $\mathcal{C} \times \mathbb{R}^{N} \subset \mathbb{R}_{+}^{N} \times \mathbb{R}^{N}$. The correspondence between $\mathcal{V}_{0}$ and $\mathcal{C}$ is provided implicitly by the centralized problem (CP), where for each $\left\{v_{\mathfrak{i}}(\cdot)\right\}_{\mathfrak{i} \in \mathcal{N}}$ we get an optimal allocation $x^{\star}$ by solving (CP) (explicitly one can solve KKT to define $x^{\star}$, together with corresponding Lagrange multipliers). This leaves the designer with the task of designing the message space and the contract.

The message space for our mechanism is $\mathcal{S}=\times_{i \in \mathcal{N}} \mathcal{S}_{i}$ with $\mathcal{S}_{i}=\left(d_{i},+\infty\right) \times \mathbb{R}_{+}^{L_{i}}$ where messages from agents are of the form $s_{i}=\left(y_{i}, p_{i}\right)$ with $p_{i}=\left(p_{i}^{l}\right)_{l \in \mathcal{L}_{i}}$ and the total message is denoted by $s=\left(s_{i}\right)_{i \in \mathcal{N}}=(y, P)$ with $y=\left(y_{i}\right)_{i \in \mathcal{N}}$ and $P=\left(p_{i}\right)_{i \in \mathcal{N}}$. The message $s_{i}=\left(y_{i}, p_{i}\right)$ is to be interpreted as follows: $y_{i}$ is the level of demand from agent $i$ and $p_{i}$ is the vector of prices that he believes everyone else should pay for the respective constraints. The contract $h: S \rightarrow \mathbb{R}_{+}^{N} \times \mathbb{R}^{N}$ will specify allocation and taxes for all agents based on the message s, i.e., $h(s)=\left(h_{x, i}(s), h_{t, i}(s)\right)_{i \in \mathcal{N}}$.

This contract along with agents' utilities will give rise to a one-shot game $\mathfrak{G}=$ $\left(\mathcal{N}, \times_{i \in \mathcal{N}} \mathcal{S}_{i},\left\{\widehat{u}_{i}\right\}_{i \in \mathcal{N}}\right)$ between agents in $\mathcal{N}$ where action sets are $\mathcal{S}_{i}$ and utilities are

$$
\widehat{u}_{i}(s)=u_{i}(x, t)=v_{i}\left(x_{i}\right)-t_{i}=v_{i}\left(h_{x, i}(s)\right)-h_{t, i}(s) .
$$

Information assumptions We assume that for any agent $i, v_{i}(\cdot)$ is his private information. The mechanism designer doesn't know $\left\{v_{i}(\cdot)\right\}_{i \in \mathcal{N}}$ but knows the set $\nu_{0}$ to which they belong. Also the constraints $\left(\mathrm{C}_{1}\right)$ and $\left(\mathrm{C}_{2}\right)$ in ( $\left.\mathrm{CP}\right)$ (along with the assumptions) are common knowledge i.e. known to agents and the mechanism designer.

We say that the mechanism fully implements the centralized problem (CP) if all Nash equilibria of the induced game $\mathfrak{G}$ correspond to the unique allocation $\chi^{\star}$ - solution of (CP), and additionally individual rationality is satisfied i.e. agents are weakly betteroff participating in the contract at equilibrium than not participating at all. 


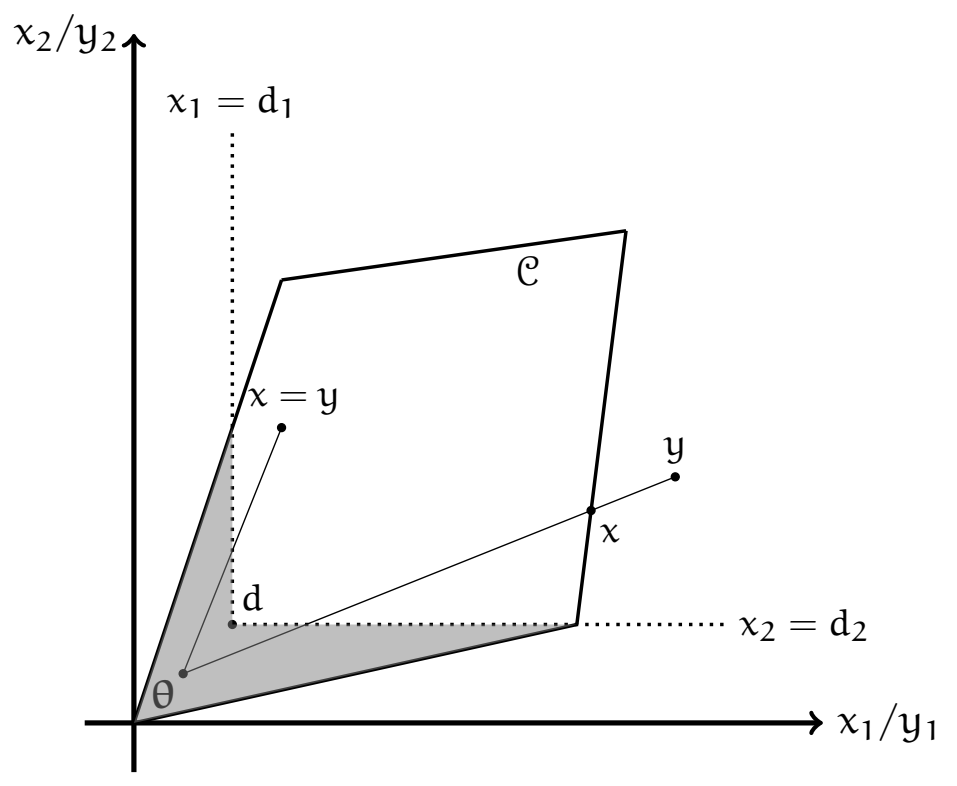

Figure 1: An illustration of the allocation for $\mathrm{N}=2$

\subsection{Allocation}

We first describe the allocation in the case where the constraints in $\left(\mathrm{C}_{2}\right)$ do not have any effective degeneracy i.e. equality constraints. This distinction is based on whether the feasible set $\mathcal{C}$ has a proper interior or not. Clearly when there are no equality constraints, the constraint set will have an interior.

For allocation in this case, we first choose a point $\theta$ in the interior of the feasible set such that

$$
\theta \in \operatorname{int}(\mathcal{C}) \cap \times_{i=1}^{N}\left(0, d_{i}\right)
$$

Note that we can guarantee the existence of $\theta$ since by assumption (A3), $\underline{0} \in \mathcal{C}$ and clearly $\mathcal{C}$ being intersection of half-planes, is a convex set. Since $d$ can be made arbitrarily close to $\underline{0}$, the same holds for $\theta$ as well.

Before formally defining the allocation, we define it informally with the help of Fig. 1. For any demand $y \in \mathcal{S}_{y} \triangleq x_{i=1}^{N}\left(d_{i},+\infty\right)$, the allocation $x$ will be equal to $y$ if $y$ is inside the feasibility region $\mathcal{C}$. Otherwise the allocation will be the intersection point between the boundary of the feasibility region $\mathcal{C}$ and the line joining $\theta$ with $y$ (that intersection point which lies between $\theta$ and $y$ ). The figure shows two different possible $y$ 's and their corresponding allocation $x$. The shaded region represents that part of $\mathcal{C}$ that can never be allocated. Note that since $\|\mathrm{d}\|,\|\theta\| \approx 0$ this is a very small region and thus it doesn't 
significantly affect the generality of results presented in this paper. Also this can be seen as a partial justification for why assumption (A2) was needed.

Formally, in case of feasibility set $\mathcal{C}$ not having any degeneracy, for a demand $y \in \mathcal{S}_{y}$, the allocation $x$ generated by the contract is

$$
x= \begin{cases}y & \text { if } y \in \mathcal{C} \\ y_{0} & \text { if } y \notin \mathcal{C},\end{cases}
$$

where $y_{0}$ is the point on the boundary of $\mathcal{C}$ which is also on the line joining $\theta$ with $y$. Explicitly, if the above intersection happens on the hyperplane $\mathcal{F}_{l}=\left\{A_{l}^{\top} x=c_{l}\right\}$ then we can express $y_{0}$ as

$$
y_{0}-\theta=\alpha_{0}(y-\theta) \quad \text { with } \quad \alpha_{0}=\frac{c_{l}-A_{l}^{\top} \theta}{A_{l}^{\top}(y-\theta)}
$$

The above allocation mapping is an extension of generalized proportional allocation idea (see [Sinha and Anastasopoulos, 2013] and [Sinha and Anastasopoulos, 2014]), but modified in accordance with the generality of the problem (CP) and also so that points in the interior of $\mathcal{C}$ are covered as well. It is easy to verify in above that for $y \notin \mathcal{C}$, because of the way proportional allocation is defined, the expression for $\alpha_{0}$ above is well-defined and positive. Also, if one extends the definition of $y_{0}$ to the boundary i.e $y \in \partial \mathcal{C}$ then it is easy to see that $y_{0}=y$ and $\alpha_{0}=1$.

Another useful explicit way of defining $\alpha_{0}$ is as follows.

$$
\alpha_{0}=\min _{\substack{l \in \mathcal{L} \\ \alpha_{0}^{\leftarrow}>0}} \alpha_{0}^{l} \quad \text { with } \quad \alpha_{0}^{l}=\frac{c_{l}-A_{l}^{\top} \theta}{A_{l}^{\top}(y-\theta)} \quad \forall l \in \mathcal{L}
$$

In the above, $c_{l}-A_{l}^{\top} \theta>0$ for all $l \in \mathcal{L}$ (because $\theta \in \operatorname{int}(\mathcal{C})$ ), but $A_{l}^{\top}(y-\theta)$ might be positive, negative or zero (since we are considering all $l \in \mathcal{L}$ and not restricting ourselves to appropriate regions as before). The purpose of taking minimum of $\alpha_{0}^{l}$ over positive ones is to find the "innermost" (or closest) constraint to $\theta$ in the (positive) direction of $y-\theta$.

Now we turn our attention to the case of degenerate feasible set. Since we are mainly interested in dealing with problems of (local) public goods nature, we define an alternate characterization of the constraint set only for those cases 4 . We can rewrite constraints from $\left(C_{2}\right)$ to explicitly account for equalities. For this consider disjoint sets of agents

\footnotetext{
${ }^{4}$ This generalizes in a straightforward way.
} 
$\left(\mathcal{N}_{k}\right)_{k \in \mathcal{K}}$, where $\mathcal{K}=\{1, \ldots, K\}$, such that $\mathcal{N}=\sqcup_{k \in \mathcal{K}} \mathcal{N}_{k}$, and the partition $\left(\mathcal{N}_{k}\right)_{k \in \mathcal{K}}$ of $\mathcal{N}$ serves the purpose of grouping agents by locality. Thus the rewritten constraints are

$$
\begin{gathered}
x_{i}=x_{j} \quad \forall i, j \in \mathcal{N}_{k}, \forall k \in \mathcal{K} \\
A_{l}^{\top} x \leqslant c_{l} \quad \forall l \in \widetilde{\mathcal{L}} \subseteq \mathcal{L},
\end{gathered}
$$

where $\widetilde{\mathcal{L}}$ is the subset of original constraints - the ones remaining after equality constraints have been separated. Without stating formally, we assume that the constraints in $\left(\mathrm{C}_{2 \mathrm{~b}}\right)$ do not introduce any further degeneracy. Now one can consider a further refinement of above to state the constraints only in terms of free variables and thus containing only inequality constraints. For this define $\widetilde{x}=\left(x_{j_{k}}\right)_{k \in \mathcal{K}}$ where $j_{k}$ represents the agent with lowest index from $\mathcal{N}_{k}$ (to make the representation unique). Clearly this is exactly the set of free variables from above and we can rewrite $\left(C_{2}\right)$ as

$$
\widetilde{A}_{l}^{\top} \widetilde{x} \leqslant c_{l} \quad \forall l \in \widetilde{\mathcal{L}},
$$

with $\widetilde{A}_{l} \in \mathbb{R}^{K}$ being derived from $A_{l}$ by using $\left(C_{2 a}\right)$ explicitly. Recall that from assumption (A4) we had at least 2 agents on each constraint, in cases such as above we extend that to have at least 2 agents per constraint in the above definition. We denote by $\widetilde{\mathcal{C}} \subset \mathbb{R}_{+}^{K}$ the effective polytope in the $\widetilde{x}$-space, created by the constraints in $\left(\mathrm{C}_{2 \mathrm{c}}\right)$.

Coming back to allocation, for demand $y \in \mathcal{S}_{y}$ we first create modified demand $\widetilde{y} \in$ $\times_{i=1}^{K}\left(\widetilde{d}_{i},+\infty\right) \subset \mathbb{R}_{+}^{K}$ as follows:

$$
\widetilde{y}_{k}=\frac{1}{N_{k}} \sum_{i \in \mathcal{N}_{k}} y_{i} \forall k \in \mathcal{K},
$$

i.e. averaging quoted y's from each group to get one representative demand. Then proportional allocation, as above, is performed on $\widetilde{y}$ and the feasibility set $\widetilde{\mathcal{C}}$ to get $\widetilde{x} \in \widetilde{\mathcal{C}}$ (note that $\widetilde{\theta}$ - the restriction of $\theta$ to appropriate coordinates, will be used in place of $\theta$ ). This proxy allocation $\widetilde{x}$ is finally converted back, using $\left(\mathrm{C}_{2 \mathrm{a}}\right)$, to get $x \in \mathcal{C}$.

\subsection{Taxes}

For any agent $i$, we define his tax $t_{i}$ as

$$
\begin{gathered}
t_{i}=\sum_{l \in \mathcal{L}_{i}} t_{i}^{l} \\
t_{i}^{l}=A_{l i} x_{i} \bar{p}_{-i}^{l}+\left(p_{i}^{l}-\bar{p}_{-i}^{l}\right)^{2}+\eta \bar{p}_{-i}^{l} p_{i}^{l}\left(c_{l}-A_{l}^{\top} x\right)^{2}
\end{gathered}
$$




$$
\bar{p}_{-i}^{l} \triangleq \frac{1}{N^{l}-1} \sum_{\substack{j \in \mathcal{N}^{l} \\ j \neq i}} p_{j}^{l}
$$

With $\eta>0$ being a positive constant.

In the results section we will see how the various terms in the tax above represent different KKT conditions. Note for example the price-taking nature that $A_{l i} x_{i} \bar{p}_{-i}^{l}$ induces; here the agent is supposed to pay for his allocation but the price at which he has to pay is decided by others.

\section{Equilibrium Results}

In this section we state and prove lemmas that will give us the desired full implementation property for the mechanism defined in Section 3. Later in this section, the presented mechanism will be modified slightly to get an additional property of SBB at NE.

The main result of full implementation would require us to prove that all pure strategy $\mathrm{NE}$ of game $\mathfrak{G}$ result in allocation $x^{\star}$ - the unique solution of (CP), and to show individual rationality. The method for proving this result is as follows: firstly we show (Lemmas 4.1. 4.5) that for any pure strategy NE the corresponding allocation and quoted prices must satisfy the KKT conditions from Section 2.4. Since by assumptions KKT conditions are both necessary and sufficient, this would mean that if pure strategy NE exist (unique or multiple), the corresponding allocation would have to be the solution of (CP) with quoted prices as the optimal Lagrange multipliers. Then we show existence (in Lemma 4.6) by explicitly writing out the messages that achieve the solution of (CP) and showing that there are no profitable unilateral deviations from those. Finally individual rationality will be shown (in Lemma 4.7).

For Lemma 4.5, we would need to distinguish between various cases arising from the general form of (다 $)$. We will make specific assumptions when necessary.

Lemma 4.1 (Primal Feasibility). For any message $s=(y, P) \in \mathcal{S}$ with corresponding allocation $x$, we have $x \in \mathcal{C}$ i.e. allocation is always feasible.

Proof. Consider the case where $\mathcal{C}$ doesn't consist of equality constraints i.e. is not degenerate. For $y \in \mathcal{C}$, feasibility of allocation $x$ is obvious. For $y \notin \mathcal{C}$, the allocation is chosen on the boundary of $\mathcal{C}$, hence it is feasible as well (note that $\mathcal{C}$ is closed).

In case when $\mathcal{C}$ is degenerate, the dummy allocation $\widetilde{x}$ is feasible w.r.t. $\widetilde{\mathcal{C}}$ using the above argument. But since the allocation $x$ is produced from $\widetilde{x}$ whilst satisfying the equal- 
ity constraints, this means that $x$ satisfies both the inequality and equality constraints and hence is feasible.

Lemma 4.2 (Equal Prices). At any $N E \mathrm{~s}=(\mathrm{y}, \mathrm{P})$ of the game $\mathfrak{G}, \forall \mathrm{l} \in \mathcal{L}$ and $\forall$ $i \in \mathcal{N}^{l}$, we have $p_{i}^{l}=p^{l}$ i.e. all agents on a constraint quote the same price for that constraint.

Proof. Suppose there exists a constraint $l \in \mathcal{L}$ such that $\left(p_{j}^{l}\right)_{j \in \mathcal{N}^{l}}$ are not all equal. Then there exists an agent $i \in \mathcal{N}^{l}$ such that $p_{i}^{l}>\bar{p}_{-i}^{l}$ (this is clear from definition in (17c)).

Consider the downwards price deviation $p_{i}^{l^{\prime}}=\bar{p}_{-i}^{l} \geqslant 0$ for this agent (whilst keeping all other values in $s_{i}$ the same). The difference in utility will be

$$
\begin{aligned}
\Delta \widehat{u}_{i}= & \widehat{u}_{i}\left(s_{i}^{\prime}, s_{-i}\right)-\widehat{u}_{i}\left(s_{i}, s_{-i}\right)=-\left(p_{i}^{l^{\prime}}-\bar{p}_{-i}^{l}\right)^{2}+\left(p_{i}^{l}-\bar{p}_{-i}^{l}\right)^{2} \\
& -\eta \bar{p}_{-i}^{l} p_{i}^{l^{\prime}}\left(c_{l}-A_{l}^{\top} x\right)^{2}+\eta \bar{p}_{-i}^{l} p_{i}^{l}\left(c_{l}-A_{l}^{\top} x\right)^{2} \\
= & \underbrace{\left(p_{i}^{l}-\bar{p}_{-i}^{l}\right)^{2}}_{>0}+\eta \bar{p}_{-i}^{l}(\underbrace{\left(p_{i}^{l}-\bar{p}_{i}^{l}\right.}_{>0})\left(c_{l}-A_{l}^{\top} x\right)^{2}>0
\end{aligned}
$$

Hence such a unilateral deviation is profitable. Therefore at $\mathrm{NE}$, all quoted prices for any constraint have to be the same.

Since we have shown that there is a common price $p^{l}$ for any constraint $l \in \mathcal{L}$, here

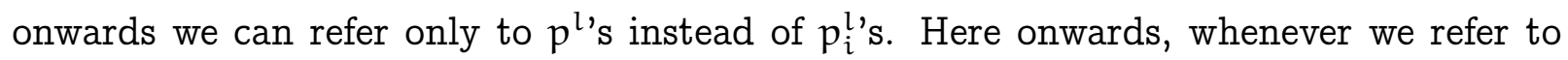
quoted price $P$, it is to be interpreted as $\left(p^{l}\right)_{l \in \mathcal{L}}$.

Lemma 4.3 (Dual Feasibility). For any $l \in \mathcal{L}, p^{l} \geqslant 0$.

Proof. This is obvious since quoted prices are all non-negative.

Lemma 4.4 (Complimentary Slackness). For any $N E \mathrm{~s}=(\mathrm{y}, \mathrm{P})$ of game $\mathfrak{G}$ with corresponding allocation $x$, we have for any $l \in \mathcal{L}$

$$
p^{l}\left(c_{l}-A_{l}^{\top} x\right)=0
$$

Proof. Suppose there exists a constraint $l \in \mathcal{L}$ such that, at NE, $p^{l}>0$ and $c^{l}>A_{l}^{\top} x$. We will take a downwards price deviation $p_{i}^{l^{\prime}}=p^{l}-\varepsilon>0$ for any agent $i \in \mathcal{N}^{l}$. Again we can write the difference in utility as

$$
\begin{gathered}
\Delta \widehat{\mathrm{u}}_{i}=-\left(\mathrm{p}_{\mathrm{i}}^{\mathrm{l}^{\prime}}-\overline{\mathrm{p}}_{-\mathrm{i}}^{\mathrm{l}}\right)^{2}+0-\eta \bar{p}_{-i}^{\mathrm{l}} \mathrm{p}_{i}^{\mathrm{l}^{\prime}}\left(\mathrm{c}_{l}-A_{l}^{\top} x\right)^{2}+\eta \overline{\mathrm{p}}_{-i}^{\mathrm{l}} p_{i}^{\mathrm{l}}\left(\mathrm{c}_{l}-A_{l}^{\top} x\right)^{2} \\
=-(-\varepsilon)^{2}+\eta p^{\mathrm{l}}(\varepsilon)\left(\mathrm{c}_{l}-A_{l}^{\top} x\right)^{2}=-\varepsilon(\varepsilon-a)
\end{gathered}
$$

with $a=\eta p^{l}\left(c_{l}-A_{l}^{\top} x\right)^{2}>0$. Now choosing $0<\varepsilon<\min \left(a, p^{l}\right)$ this can be made into a profitable deviation. 
Next we have the lemma for the stationarity property from KKT. For this we have to make assumptions on the feasible set $\mathcal{C}$.

$$
\widetilde{A}_{l} \in \mathbb{R}_{+}^{K} \forall l \in \widetilde{\mathcal{L}}
$$

where $\widetilde{A}_{l}$ was defined in $\left(\mathrm{C}_{2 \mathrm{c}}\right)$ (Section 3.1).

The above clearly translates into $A_{l} \in \mathbb{R}_{+}^{N} \forall l \in \mathcal{L}$ in case there is no degeneracy. But when there is degeneracy, the above assumption states that the effective polytope $\widetilde{\mathcal{C}}$ in the $\tilde{x}$-space has faces whose outward normals are pointing away from the origin.

Lemma 4.5 (Stationarity). At any $N E \mathrm{~s}=(\mathrm{y}, \mathrm{P})$ of game $\mathfrak{G}$ with corresponding allocation $x$, we have for any agent $i \in \mathcal{N}$,

$$
v_{i}^{\prime}\left(x_{i}\right)=\sum_{l \in \mathcal{L}_{i}} A_{l i} p^{l}
$$

Proof. For any agent $i \in \mathcal{N}$ we can write

$$
\frac{\partial \widehat{u}_{i}(s)}{\partial y_{i}}=\left(v_{i}^{\prime}\left(x_{i}\right)-\frac{\partial t_{i}}{\partial x_{i}}\right) \frac{\partial x_{i}}{\partial y_{i}}
$$

Note that $\beta \equiv \partial x_{i} / \partial y_{i}$ isn't always defined, since the allocation is continuous but only piecewise differentiable for $y \notin \mathcal{C}$. But right and left derivatives are always defined. Noting that $v_{i}^{\prime}\left(x_{i}\right)-\frac{\partial t_{i}}{\partial x_{i}}=0$ is equivalent to (22) (using previous lemmas characterizing $\mathrm{NE}$ ), it will be sufficient for us if we show that $\beta>0$. Since then without making $v_{i}^{\prime}\left(x_{i}\right)-\frac{\partial t_{i}}{\partial x_{i}}=0$ there is always an upwards or downwards deviation in $y_{i}$ to make agent $i$ strictly better-off.

For $y \in \mathcal{C}$ we have $x_{i}=y_{i}$, therefore $\beta=1 \neq 0$. Otherwise, first consider the case where $\mathcal{C}$ is non-degenerate. Then

$$
\begin{gathered}
x_{i}=\theta_{i}+\alpha_{0}\left(y_{i}-\theta_{i}\right) \quad \text { with } \quad \alpha_{0}=\frac{c_{l}-A_{l}^{\top} \theta}{A_{l}^{\top}(y-\theta)} \\
\frac{\partial x_{i}}{\partial y_{i}}=\alpha_{0}+\left(y_{i}-\theta_{i}\right) \frac{\partial \alpha_{0}}{\partial y_{i}} \quad \text { with } \quad \frac{\partial \alpha_{0}}{\partial y_{i}}=\frac{-\alpha_{0} A_{l i}}{A_{l}^{\top}(y-\theta)} \\
\Rightarrow \frac{\partial x_{i}}{\partial y_{i}}=\alpha_{0}\left(1-\frac{A_{l i}\left(y_{i}-\theta_{i}\right)}{A_{l}^{\top}(y-\theta)}\right)=\alpha_{0}\left(\sum_{j \in \mathcal{N}^{l} \backslash\{i\}} A_{l j}\left(y_{j}-\theta_{j}\right)\right)
\end{gathered}
$$

Noting that $\alpha_{0}>0$ and that due to assumption (A4) there are always at least two agents on any constraint, clearly the above is positive ( since $\left.\theta_{j}<d_{j}<y_{j} \forall j\right)$.

In the case when $\mathcal{C}$ does have equality constraints, note that allocations are created by composing the maps $\mathrm{y} \stackrel{\mathrm{A}}{\mapsto} \widetilde{\mathrm{y}} \stackrel{\mathrm{B}}{\mapsto} \widetilde{\mathrm{x}} \stackrel{\mathrm{C}}{\mapsto} \mathrm{x}$ where $\mathrm{B}$ is proportional allocation on the set $\widetilde{\mathcal{C}}$ 
and $A, C$ are linear with positive coefficients 5 . Thus using the fact that for proportional allocation we have $\beta>0$ (from above) and that $A, C$ have positive coefficients, we clearly have $\beta>0$ here as well.

This completes the necessary part of our proof. The argument in the previous Lemmas applied to pure strategy NE if there exited any. Now we will prove the existence of pure strategy NE which will give the optimal allocation $x^{\star}$.

Lemma 4.6 (Existence). For game $\mathfrak{G}$, there exists $N E \mathrm{~s}=(\mathrm{y}, \mathrm{P}) \in \mathcal{S}$ such that the corresponding allocation $x$ and prices $\left(\mathrm{p}^{l}\right)_{\mathrm{l} \in \mathcal{L}}$ satisfy the KKT conditions as $\chi^{\star}$ and $\left(\lambda_{l}^{\star}\right)_{l \in \mathcal{L}}$, respectively.

Proof. This proof will be done in two separate parts: first we will show that there exists $s \in \mathcal{S}$ such that the allocation through the contract is $\chi^{\star}$ and prices are Lagrange multipliers $\left(\lambda_{l}^{\star}\right)_{l \in \mathcal{L}}$. Secondly, we will show that for all claimed NE points, there doesn't exist a unilateral deviation that is profitable.

Existence of prices that match Lagrange multipliers $\lambda_{l}^{\star}$ is obvious, since agents can quote any price in $\mathbb{R}_{+}$and dual feasibility says that $\lambda_{l}^{\star} \geqslant 0$. For allocation to be the same as $x^{\star}$, notice that due to assumption (A2) all possible solutions $x^{\star}$ lie in the set $y \triangleq \mathcal{C} \cap \mathcal{S}_{y}$ with $\mathcal{S}_{y}=\times_{i=1}^{N}\left(d_{i},+\infty\right)$. Quoted demand $y$ can be anywhere in the set $\mathcal{S}_{y}$ and for $y \in \mathcal{C}$ (i.e. $y \in y$ ) the allocation is $x=y$ and therefore each point $x \in y$ is achievable as allocation by quoting the same point $y=x \in y$. (However also note that points on the boundary of $y$ are achievable as allocation by many $y$ 's outside $y$ as well.)

Now we will check for unilateral profitable deviations. When quoted demand creates allocation as $x^{\star}$ and the quoted prices are equal and equal to $\lambda^{\star}$, the utility for any agent $i$ is

$$
\widehat{u}_{i}(s)=v_{i}\left(x_{i}^{\star}\right)-x_{i}^{\star} \sum_{l \in \mathcal{L}_{i}} A_{l i} \lambda_{l}^{\star}
$$

Due to the fact that $f(x)=v_{i}(x)-x \sum_{l \in \mathcal{L}_{i}} A_{l i} \lambda_{l}^{\star}$ is strictly concave and $f^{\prime}\left(x_{i}^{\star}\right)=0$ (Stationarity) we can conclude that $x_{i}^{\star}$ is a global maximizer of $f$. With this we have

$$
\begin{gathered}
\widehat{u}_{i}(s)=v_{i}\left(x_{i}^{\star}\right)-x_{i}^{\star} \sum_{l \in \mathcal{L}_{i}} A_{l i} \lambda_{l}^{\star} \geqslant v_{i}\left(x_{i}\right)-x_{i} \sum_{l \in \mathcal{L}_{i}} A_{l i} \lambda_{l}^{\star} \\
\geqslant v_{i}\left(x_{i}\right)-x_{i} \sum_{l \in \mathcal{L}_{i}} A_{l i} \lambda_{l}^{\star}-\sum_{l \in \mathcal{L}_{i}}\left(p_{i}^{l}-\lambda_{l}^{\star}\right)^{2}-\sum_{l \in \mathcal{L}_{i}} \eta p_{i}^{l} \lambda_{l}^{\star}\left(c_{l}-A_{l}^{\top} x\right)^{2}
\end{gathered}
$$

The above is true for any $\left(x_{j}\right)_{j \in \mathcal{N}}$ and $\left(p_{i}^{l}\right)_{l \in \mathcal{L}_{i}}$ non-negative and final inequality holds because the additional terms are non-positive. Now any unilateral deviation $\left(s_{i}^{\prime}, s_{-i}\right)$

\footnotetext{
${ }^{5}$ Since they consist of averaging, (16), and assigning same value to multiple positions.
} 
from agent $i$ will result in utility for agent $i$ which has the form as in (26b). Hence we have proved that with unilateral deviations from messages that have allocation $x^{\star}$ and prices $\lambda^{\star}$, the corresponding agent can never be strictly better off.

Lemma 4.7 (Individual Rationality). For any $N E \mathrm{~s}=(\mathrm{y}, \mathrm{P})$ of game $\mathfrak{G}$ with corresponding allocation $x$ and taxes $t$, we have for any $i \in \mathcal{N}$

$$
u_{i}(x, t) \geqslant u_{i}(0,0)
$$

Proof. Recall that $\mathfrak{u}_{\mathfrak{i}}(x, t)=v_{i}\left(x_{i}\right)-t_{i}$. For any agent $i \in \mathcal{N}$, define

$$
f(z) \triangleq v_{i}(z)-z \sum_{l \in \mathcal{L}_{i}} A_{l i} p^{l}
$$

Note that $u_{i}(0,0)=v_{i}(0)=f(0)$ and at NE $u_{i}(x, t)=v_{i}\left(x_{i}\right)-x_{i} \sum_{l \in \mathcal{L}_{i}} A_{l i} p^{l}=f\left(x_{i}\right)$ (also recall that except the first term, all other tax terms go to zero at NE, refer (17b)).

By stationarity property we know $f^{\prime}\left(x_{i}\right)=v_{i}^{\prime}\left(x_{i}\right)-\sum_{l \in \mathcal{L}_{i}} A_{l i} p^{l}=0$ and by assumption (A1) it is clear that $f(\cdot)$ is strictly concave. Therefore $f^{\prime}(y)>0$ for $0<y<x_{i}$ and we can claim by mean value theorem that $f\left(x_{i}\right)>f(0)$.

Theorem 4.8 (Full Implementation). All Nash equilibria $s=(y, P)$ of the game $\mathfrak{G}$ have the same corresponding allocation $x$. Furthermore, this allocation is identical to the unique solution of $(\mathrm{CP})$ i.e. $x=x^{\star}$. Also, all agents are weakly better-off at equilibrium than by not participating at all.

Proof. Thus from Lemma 4.6 there exists at least one pure strategy NE. From Lemma4.1. 4.5, allocation $x$ at any NE has to satisfy KKT conditions and knowing that KKT conditions are necessary and sufficient for optimality, it is clear that $x=x^{\star}$. Finally, individual rationality was shown in Lemma 4.7 and with this we have our full implementation result.

\subsection{Strong Budget Balance (at equilibrium)}

In this section we present a modification in the above mechanism so that in addition to above properties we also have SBB, i.e., $\sum_{i \in \mathcal{N}} t_{i}=0$ at those equilibria where $y \in \mathcal{C}$.

The message space and allocation $x$ is exactly same as before, the only modification will be to the taxes. Whenever the quoted demand is feasible, we have $x=y$. So from (17a), (17b) and previous lemmas we can write total tax at $\mathrm{NE}$ as

$$
\sum_{i \in \mathcal{N}} t_{i}=\sum_{i \in \mathcal{N}} \sum_{l \in \mathcal{L}_{i}} t_{i}^{l}=\sum_{l \in \mathcal{L}} \sum_{i \in \mathcal{N}^{l}} t_{i}^{l}=\sum_{l \in \mathcal{L}} \sum_{i \in \mathcal{N}^{l}} A_{l i} y_{i} p^{l}
$$


To achieve SBB, our approach is to redistribute the above total tax amongst all the agents so that the amount of money received by an agent will not be controlled by that agent i.e. it will be only a function of $y, p$ quoted by other agents. This will ensure that strategic decisions are still same as before and thus we would continue to have all the previous properties (Lemmas 4.144.6). Furthermore, we will redistribute taxes per constraint. So for any constraint $l \in \mathcal{L}, \sum_{i \in \mathcal{N}^{l}} t_{i}^{l}$ will be redistributed only amongst agents in $\mathcal{N}^{l}$. After appropriately redistributing the taxes, we will check individual rationality at equilibrium once again since the total tax paid by an agent might have a different value now. The reason we present SBB as a side result is that one can always use such a redistribution technique and thus SBB generally reduces to appropriate algebraic manipulation and can usually be added later on in the mechanism (for problems of these types).

Let $T_{i}^{l}$ be the new tax where analogously the old tax (from (17b)) was $t_{i}^{l}$. Then the total tax for any agent $i$ is $T_{i}=\sum_{l \in \mathcal{L}_{i}} T_{i}^{l}$. From above discussion, what we want is

$$
T_{i}^{l}=t_{i}^{l}-f_{i}^{l}\left(\left(y_{j}, p_{j}^{l}\right)_{\substack{j \in \mathcal{N}^{l} \\ j \neq i}}\right)
$$

for any $i \in \mathcal{N}$ and $l \in \mathcal{L}_{i}$. The function $f_{i}^{l}$ will be defined below.

For $l \in \mathcal{L}$ such that $N^{l} \geqslant 3$, in case where $A_{l i} \geqslant 0 \forall i \in \mathcal{N}^{l}$, we have

$$
f_{i}^{l} \equiv f_{i, 1}^{l}=\frac{1}{N^{l}-2} \sum_{\substack{j \in \mathcal{N}^{l} \\ j \neq i}} A_{l j} y_{j}\left(\bar{p}_{-i}^{l}-\frac{p_{j}^{l}}{N^{l}-1}\right)
$$

when there are negative coefficients in $A_{l i}$, we can define $f_{i}^{l}$ in the same manner as above, except that coefficients for those $j$ that are involved in an equality constraint will be (scaled)6 $\widetilde{A}_{l j}$ instead of $A_{l j}$.

For $l \in \mathcal{L}$ such that $N^{l}=2$ (assuming $\mathcal{N}^{l}=\{i, j\}$ ), we divide the redistribution into 2 cases: first we consider the case $A_{l i}, A_{l j} \geqslant 0$, for this we have

$$
f_{i}^{l} \equiv f_{i, 1}^{l}=A_{l j} y_{j} p_{j}^{l} .
$$

If the coefficients $A_{l i}$ are not positive then we are essentially into the degenerate case, where $\left|A_{l i}\right|=\left|A_{l j}\right|=1$ with opposite signs. For this we have $f_{i}^{l} \equiv f_{i, 1}^{l}=0$ and same for agent $j$.

With the modified tax we will denote the new game as $\mathfrak{G}^{\prime}$.

\footnotetext{
${ }^{6}$ the scaling factor being the inverse of the number of agents on $l$ that are involved in constraints that make their allocation equal.
} 
Lemma 4.9 (Strong Budget Balance at NE). For the game $\mathfrak{G}^{\prime}$, at all $N E \mathrm{~s}=(\mathrm{y}, \mathrm{P})$ where $y \in \mathcal{C}$ we have

$$
\sum_{i \in \mathcal{N}} T_{i}=\sum_{i \in \mathcal{N}} \sum_{l \in \mathcal{L}_{i}} T_{i}^{l}=0
$$

Proof. Recall that due to Lemmas 4.2 and 4.4 , at $\mathrm{NE}$ we have

$$
\sum_{i \in \mathcal{N}} t_{i}=\sum_{i \in \mathcal{N}} \sum_{l \in \mathcal{L}_{i}} t_{i}^{l}=\sum_{l \in \mathcal{L}} \sum_{i \in \mathcal{N}^{l}} t_{i}^{l}=\sum_{l \in \mathcal{L}} \sum_{i \in \mathcal{N}^{l}} A_{l i} y_{i} p^{l}
$$

For completing the proof, it will be sufficient to show $\sum_{i \in \mathcal{N}^{l}} t_{i}^{l}=\sum_{i \in \mathcal{N}^{l}} f_{i}^{l}$ for any $l \in \mathcal{L}$, at $N E$. Let's begin with links with $N^{l}=2\left(\right.$ where $\left.\mathcal{N}^{l}=\{i, j\}\right)$. In case $A_{l i}, A_{l j} \geqslant 0$ the total payment is

$$
f_{i}^{l}+f_{j}^{l}=A_{l j} y_{j} p_{j}^{l}+A_{l i} y_{i} p_{i}^{l}=\sum_{k \in \mathcal{N}^{l}} A_{l k} y_{k} p^{l}
$$

In the degenerate case, the tax paid by agents $i, j$ at $N E$ is $y_{i} p^{l}, y_{j} p^{l}$ with opposite signs. But note that $y_{i}=y_{j}$ at $N E$, since we are in the degenerate case. Hence the total tax is zero, which is the same as $f_{i}^{l}+f_{j}^{l}$. Now for other links,

$$
\begin{gathered}
\sum_{i \in \mathcal{N}^{l}} \frac{1}{N^{l}-2} \sum_{\substack{j \in \mathcal{N}^{l} \\
j \neq i}} A_{j l} y_{j}\left(\bar{p}_{-i}^{l}-\frac{p_{j}^{l}}{N^{l}-1}\right)=\frac{1}{N^{l}-2} \sum_{i \in \mathcal{N}^{l}} \sum_{\substack{j \in \mathcal{N}^{l} \\
j \neq i}} A_{j l} y_{j}\left(\bar{p}_{-j}^{l}-\frac{p_{i}^{l}}{N^{l}-1}\right) \\
=\frac{N^{l}-1}{N^{l}-2} \sum_{j \in \mathcal{N}^{l}} A_{j l} y_{j} \bar{p}_{-j}^{l}-\frac{1}{N^{l}-2} \sum_{\substack { j \in \mathcal{N}^{l} \\
\begin{subarray}{c}{i \in \mathcal{N}^{l} \\
i \neq j{ j \in \mathcal { N } ^ { l } \\
\begin{subarray} { c } { i \in \mathcal { N } ^ { l } \\
i \neq j } }\end{subarray}} A_{j l} y_{j} \frac{p_{i}^{l}}{N^{l}-1} \\
=\frac{N^{l}-1}{N^{l}-2} \sum_{j \in \mathcal{N}^{l}} A_{j l} y_{j} \bar{p}_{-j}^{l}-\frac{1}{N^{l}-2} \sum_{j \in \mathcal{N}^{l}} A_{j l} y_{j} \bar{p}_{-j}^{l}=\sum_{j \in \mathcal{N}^{l}} A_{j l} y_{j} \bar{p}_{-j}^{l}=\sum_{j \in \mathcal{N}^{l}} A_{j l} y_{j} p^{l}
\end{gathered}
$$

(the proof when $A_{l j}$ are possibly negative also follows from above after explicitly using that equality constraints would make some allocations equal to each other at $\mathrm{NE}$ )

After this, we have to check individual rationality. In cases when there is no degeneracy in $\mathcal{C}$, due to assumption (A6), $A_{l i} \geqslant 0 \forall l$, i. This means that with $f_{i}^{l}$ we have redistributed taxes by paying agents back, thus the redistribution is indeed non-negative (can be seen explicitly from (31) and (32)). Thus if the NE was individually rational without this redistribution, it will continue to be so now.

For the cases involving degeneracy, for links associated with equality constraints the redistribution is zero. So individual rationality isn't affected. For other links, individual rationality follows from the argument in the previous paragraph after noting that effectively $\widetilde{A}_{l i}$ were used in place of $A_{l i}$ for defining $f_{i}^{l}$ (and by (A6), $\widetilde{A}_{l i} \geqslant 0$ ). 


\section{Off-equilibrium Results}

In this section we discuss (and prove) additional off-equilibrium properties of the mechanism. The original requirements of full implementation are only restricted to equilibrium properties. However we believe that in a realistic scenario, off-equilibrium properties are essential to justify working within a Nash-implementation framework. Nash equilibrium is an appropriate solution concept for complete information games i.e. games where it is assumed that all agents have complete knowledge about each others utility functions and also each agent knows that others know about his utility functions and so on (infinite hierarchy of beliefs). We are proposing this mechanism to be used in an informationally, and possibly physically, decentralized network setting like the Internet. The root of delving into mechanism design is that the designer doesn't have information about the utility functions, so to assume that agents themselves have all the information is impractical, especially for communications settings. In absence of complete information with agents, Nash implementation has still been used in literature - the justification being online or offline learning. In this formulation, before playing the actual game, agents participate in a multi-round learning process. After the agents have learnt about each others utilities (or more specifically the equilibrium action) they play the actual one-shot game.

In order to make the above learning model practical, there have to be real incentives involved for learning correctly. It is expected that while the learning process is still going on, in every round agents will quote demands and prices and receive allocation and taxes through the contract. We are interested in dealing with constrained resource allocation problems where the constraints could possibly be of a hard nature i.e. impossible to violate. An instance of this is the capacity constraint in unicast and multi-rate multicast examples. Hence if one assumes the learning model, then a necessary property for the mechanism would be that allocation is always (even off-equilibrium) feasible, since during the learning process agents will be playing off-equilibrium. To a lesser extent, if one is interested in SBB then same would have to be true of SBB as well. It is for these reasons that we introduced the proportional allocation, as a distinct feature of our mechanism, as opposed to the simple allocation used in [Healy and Mathevet, 2012], [Kakhbod and Teneketzis, 2013a], where allocation equals demand, i.e. $x=y$ everywhere.

Since the mid-90s, various learning models in Mechanism design and Game theory have been extensively studied. Readers may refer to [Healy and Mathevet, 2012] for studying how mechanism design and learning are handled together. Also one may refer to [Young, 2004], [Fudenberg, 1998], [Tembine, 2012], [Shoham and Leyton-Brown, 2009], [Menache and Ozdaglar, 2011], [Lasaulce and Tembine, 2011] for a compendium 
of existing results regarding learning in a strategic set-up.

Now we extend the modification from Section 4.1 to achieve SBB off-equilibrium.

\subsection{SBB off-equilibrium}

Here we will define $f_{i}^{l}$ from (30) differently so that we achieve the property of $\sum_{i \in \mathcal{N}} t_{i}=0$ at all points where $y \in \mathcal{C}$, not just at NE. From the expressions in (29) as well as the description of $f_{i}^{l}$ below, it will be clear that this method of redistribution only works when there are sufficient number of agents on every link. So here we will modify assumption (A4) so that

$\left(\mathrm{A} 4^{\prime}\right)$ For any $l \in \mathcal{L}, \mathrm{N}^{l} \geqslant 5$ i.e. there are at least 5 agents on any constraint.

Also, we will only deal with the non-degenerate case where $A_{l i} \geqslant 0 \forall l$,i. The corresponding results regarding degenerate cases would involve tedious case-by-case analysis and unnecessarily complicate the analysis of what should be a straightforward addition to the mechanism.

Now $f_{i}^{l}$ is defined in three parts: $f_{i}^{l}\left(\left(y_{j}, p_{j}^{l}\right)_{\substack{j \in \mathcal{N}^{l} \\ j \neq i}}\right)=f_{i, 1}^{l}+f_{i, 2}^{l}+f_{i, 3}^{l}$. The three terms here are individually redistributing the three tax terms from (29)).

$$
\begin{gathered}
f_{i}^{l} \equiv f_{i, 1}^{l}=\frac{1}{N^{l}-2} \sum_{\substack{j \in \mathcal{N}^{l} \\
j \neq i}} A_{l j} y_{j}\left(\bar{p}_{-i}^{l}-\frac{p_{j}^{l}}{N^{l}-1}\right) \\
f_{i, 2}^{l}=\frac{N^{l}}{\left(N^{l}-1\right)^{2}\left(N^{l}-2\right)} \sum_{\substack{k>m \\
k, m \neq i}}\left(p_{k}^{l}-p_{m}^{l}\right)^{2} \\
f_{i, 3}^{l}=\eta\left(f_{i, 3 a}^{l}+f_{i, 3 b}^{l}+f_{i, 3 c}^{l}\right) \\
f_{i, 3 b}^{l}=\frac{2}{N^{l}-1} \sum_{\substack{j>q \\
j, q \neq i}}\left[\frac{1}{N^{l}-3} \sum_{\substack{k \in \mathcal{N}^{l} \\
k \neq i, j, q}} p_{j}^{l} p_{q}^{l} \phi\left(y_{k}\right)+\frac{1}{N^{l}-2} \sum_{\substack{k \in \mathcal{N}^{l} \\
k=j, q}} p_{j}^{l} p_{q}^{l} \phi\left(y_{k}\right)\right]
\end{gathered}
$$

where $\phi\left(y_{k}\right)=A_{k l}^{2} y_{k}^{2}-2 c_{l} A_{k l} y_{k}$. For $f_{i, 3 c}^{l}$ define the set $B \triangleq\{j, q\} \cap\{k, s\}$,

$$
f_{i, 3 c}^{l}=\frac{4}{N^{l}-1} \sum_{\substack{j>q \\ j, q \neq i}}\left[\frac{1}{N^{l}-4} \sum_{\substack{k>s \\ k, s \neq i \\|B|=0}} \psi+\frac{1}{N^{l}-3} \sum_{\substack{k<>s \\ k, s \neq i \\|B|=1}} \psi+\frac{1}{N^{l}-2} \sum_{\substack{k>s \\ k, s \neq i \\ k=j, s=q}} \psi\right]
$$


where $\psi=\psi\left(p_{j}^{l}, p_{q}^{l}, y_{k}, y_{s}\right)=p_{j}^{l} p_{q}^{l}\left(A_{k l} y_{k}\right)\left(A_{s l} y_{s}\right)$. It is this last expression that necessitates the assumption $\left(\mathrm{A} 4^{\prime}\right)$. We will denote the game with the above modified taxes with $\mathfrak{G}^{\prime \prime}$.

Lemma 5.1 (Strong Budget Balance on and off-NE). For the game $\mathfrak{G}^{\prime \prime}$, for all points in the message space $\mathcal{S}$ where $\mathrm{y} \in \mathcal{C}$

$$
\sum_{i \in \mathcal{N}} T_{i}=\sum_{i \in \mathcal{N}} \sum_{l \in \mathcal{L}_{i}} T_{i}^{l}=0
$$

Proof. By rearranging the above sum: $\sum_{i \in \mathcal{N}} \sum_{l \in \mathcal{L}_{i}} T_{i}^{l}=\sum_{l \in \mathcal{L}} \sum_{i \in \mathcal{N}^{l}} T_{i}^{l}$, we can see that it will be sufficient if we show that for any constraint $l \in \mathcal{L}$,

$$
\sum_{i \in \mathcal{N}^{l}} T_{i}^{l}=0 \Leftrightarrow \sum_{i \in \mathcal{N}^{l}} t_{i}^{l}=\sum_{i \in \mathcal{N}^{l}} f_{i}^{l}\left(\left(y_{j}, p_{j}^{l}\right)_{\substack{j \in \mathcal{N}^{l} \\ j \neq i}}\right)
$$

We will again show this term-by-term; we have already shown in the proof of Lemma 4.9 that the sum of $f_{i, 1}^{l}$ is equal to the sum of payment (first) term from $t_{i}^{l}$, similarly the sum of $f_{i, 2}^{l}$ and $f_{i, 3}^{l}$ will be shown to be equal to the sum of the second and third terms from $t_{i}^{l}$, respectively. Starting from the second term

$$
\begin{gathered}
\sum_{i \in \mathcal{N}^{l}}\left(p_{i}^{l}-\bar{p}_{-i}^{l}\right)^{2}=\sum_{i \in \mathcal{N}^{l}}\left(p_{i}^{l}\right)^{2}+\left(\bar{p}_{-i}^{l}\right)^{2}-2 p_{i}^{l} \bar{p}_{-i}^{l} \\
=\frac{N^{l}}{N^{l}-1} \sum_{i \in \mathcal{N}^{l}}\left(p_{i}^{l}\right)^{2}-\frac{2 N^{l}}{\left(N^{l}-1\right)^{2}} \sum_{\substack{k, m \in \mathcal{M}^{l} \\
k>m}} p_{k}^{l} p_{m}^{l} \\
=\frac{N^{l}}{\left(N^{l}-1\right)^{2}}\left[\left(N^{l}-1\right) \sum_{i \in \mathcal{N}^{l}}\left(p_{i}^{l}\right)^{2}-2 \sum_{\substack{k, m \in \mathcal{N}^{l} \\
k>m}} p_{k}^{l} p_{m}^{l}\right]=\frac{N^{l}}{\left(N^{l}-1\right)^{2}} \sum_{\substack{k, m \in \mathcal{N}^{l} \\
k>m}}\left(p_{k}^{l}-p_{m}^{l}\right)^{2}
\end{gathered}
$$

Now observing

$$
\sum_{\substack{k, m \in \mathcal{N}^{l} \\ k>m}}\left(p_{k}^{l}-p_{m}^{l}\right)^{2}=\frac{1}{N^{l}-2} \sum_{i \in \mathcal{N}^{l}} \sum_{\substack{k, m \in \mathcal{N}^{l} \\ k, m \in \mathfrak{k} \\ k, m \neq i}}\left(p_{k}^{l}-p_{m}^{l}\right)^{2}
$$

we get the equality of second terms. For the third term

$$
\sum_{i \in \mathcal{N}^{l}} \eta p_{j}^{l} \bar{p}_{-j}^{l}\left(c_{l}-\sum_{k \in \mathcal{N}^{l}} \alpha_{k}^{l} y_{k}\right)^{2}=\eta c_{l}^{2} \sum_{j \in \mathcal{N}^{l}} p_{j} \bar{p}_{-j}^{l}+\sum_{j \in \mathcal{N}^{l}} \eta p_{j} \bar{p}_{-j}^{l}\left(\sum_{k \in \mathcal{N}^{l}} \phi\left(y_{k}\right)\right)
$$




$$
+2 \eta \sum_{j \in \mathcal{N}^{l}} p_{j} \bar{p}_{-j}^{l}\left(\sum_{k>m} A_{k l} A_{m l} y_{k} y_{m}\right) \equiv \eta\left(T_{1}+T_{2}+T_{3}\right)
$$

where as before $\phi\left(y_{k}\right)=A_{k l}^{2} y_{k}^{2}-2 c_{l} A_{k l} y_{k}$. Here we will equate $T_{1}, T_{2}, T_{3}$ with the sum of $f_{i, 3 a}^{l}, f_{i, 3 b}^{l}, f_{i, 3 c}^{l}$, respectively.

$$
c_{l}^{2} \sum_{j \in \mathcal{N}^{l}} p_{j} \bar{p}_{-j}^{l}=\frac{2 c_{l}^{2}}{N^{l}-1} \sum_{k>m} p_{k}^{l} p_{m}^{l}=\frac{2 c_{l}^{2}}{\left(N^{l}-1\right)\left(N^{l}-2\right)} \sum_{i \in \mathcal{N}^{l}} \sum_{\substack{k>m \\ k, m \neq i}} p_{k}^{l} p_{m}^{l}
$$

This completes $f_{i, 3 a}$, now for $f_{i, 3 b}$

$$
\begin{gathered}
\sum_{j \in \mathcal{N}^{l}} p_{j} \bar{p}_{-j}^{l}\left(\sum_{k \in \mathcal{N}^{l}} \phi\left(y_{k}\right)\right)=\frac{2}{N^{l}-1} \sum_{j>m} \sum_{k \in \mathcal{N}^{l}} p_{j}^{l} p_{m}^{l} \phi\left(y_{k}\right) \\
=\frac{2}{N^{l}-1} \sum_{j>m}\left(\sum_{k \neq j, m} p_{j}^{l} p_{m}^{l} \phi\left(y_{k}\right)+\sum_{k=j, m} p_{j}^{l} p_{m}^{l} \phi\left(y_{k}\right)\right) \\
=\frac{2}{N^{l}-1} \sum_{i \in \mathcal{N}^{l}} \sum_{\substack{j>m \\
j, m \neq i}}\left(\frac{1}{N^{l}-3} \sum_{k \neq i, j, m} p_{j}^{l} p_{m}^{l} \phi\left(y_{k}\right)+\frac{1}{N^{l}-2} \sum_{k=j, m} p_{j}^{l} p_{m}^{l} \phi\left(y_{k}\right)\right)
\end{gathered}
$$

This completes $f_{i, 3 b}$ and finally the sum for $f_{i, 3 c}$ can be shown in the same way as above.

Hence after comparing the sum of all three terms from $t_{i}^{l}$ we have indeed proved (38).

Now just as before, we check individual rationality (for the modified game $\mathfrak{G}^{\prime \prime}$ ). Overall utility for agent $i$ after redistribution, at $\mathrm{NE}$, is

$$
\widehat{u}_{i}=v_{i}\left(x_{i}\right)-x_{i} \sum_{l \in \mathcal{L}_{i}} A_{l i} p^{l}+\sum_{l \in \mathcal{L}_{i}} f_{i, 1}^{l}+f_{i, 2}^{l}+f_{i, 3}^{l}
$$

where the last summation is calculated at NE. At NE, we have equal prices, hence from (36b) it is clear that $\sum_{l \in \mathcal{L}_{i}} f_{i, 2}^{l}$ term is zero. Since we are only dealing with the non-degenerate case in this section, it is clear from the definition of $f_{i, 1}^{l}$ (see (36a)) that $\sum_{l \in \mathcal{L}_{i}} f_{i, 1}^{l} \geqslant 0$. We know already, from Lemma 4.7, that $v_{i}\left(x_{i}\right)-x_{i} \sum_{l \in \mathcal{L}_{i}} A_{l i} p^{l}-v_{i}(0)>$ 0 , furthermore since the functions $\nu_{i}$ are strictly concave and $x_{i}>d_{i}>0$ (by assumption (A2)) we can bound the difference in above inequality by a value bigger than zero.

Now note that $f_{i, 3}^{l}$ is multiplied by a positive factor $\eta$ which is still to be chosen. Since prices $p^{l}$, demand/allocation $y_{i}$ and coefficients $A_{l i}$ are all absolutely bounded, we can always make the contribution of $\sum_{l \in \mathcal{L}_{i}} f_{i, 3}^{l}$ small enough (without having the knowledge of equilibrium values in advance) so that the overall utility in (44) is bigger than $v_{i}(0)$. Hence we have individual rationality here as well. 


\section{Generalizations}

Three quite interesting generalizations arise immediately from the set-up and analysis in this paper. The first is a case where agents have utilities based on a vector allocation rather than a scalar allocation i.e., the multiple goods scenario. The objective in this case will be written as

$$
\sum_{i \in \mathcal{N}} v_{i}\left(\underline{x}_{i}\right) \quad \text { with } \quad \underline{x}_{i} \in \mathbb{R}_{+}^{D_{i}} .
$$

Note that the assumption of strict concavity can still be made. In a communications scenario, such an example can arise if the Internet agents have utility based on throughput as well as delay or packet error rate. In the case of the local public goods problem and in particular its instance that models the interaction between wireless agents, vector allocation for agent $i$ models the power level of all users that can interfere with $i$. In this case the problem $\left(\mathrm{CP}_{1 \mathrm{pb}}\right.$ can be restated as

$$
\begin{gathered}
\max _{x} \sum_{i \in \mathcal{N}} v_{i}\left(\underline{x}_{i}\right) \\
\text { s.t. } \quad \underline{x}_{i} \in \mathbb{R}_{+}^{D_{i}} \quad \forall i \in \mathcal{N} \\
\text { s.t. } \quad E_{i}^{k \top} \underline{x}_{i}=E_{j}^{k^{\top}} \underline{x}_{j} \quad \forall i, j \in \mathcal{N}_{k}, \forall k \in \mathcal{K}
\end{gathered}
$$

where $\mathcal{N}_{k}$ as before denote localities, however they needn't be disjoint now. Note that in the vector equations (46b), multiplication by matrices $E_{i}^{k}$ accomplishes the task of selecting some coordinates from $\underline{x}_{i}$.

The second generalization is with problems which can be equivalently formulated in the form of ( $\mathrm{CP}$ ), but perhaps with the help of auxiliary variables. Consider the multi-rate multicast system, in which agents are divided into multicast groups, where agents within a multicast group communicate with exactly the same data but possibly at different quality (information rate) for each agent. This problem has both - private and public goods characteristics. Just like unicast, the limited capacity on links creates constraints on allocation here. However for saving bandwidth, only the highest demanded rate from each multicast group is transmitted on every link. This means that resources are directly shared within every group. If we index agents by a double-index $k i \in \mathcal{N}$ where $k$ represents their multicast group and $i$ is the sub-index within the multicast group $k$, we can state the optimization problem as

$$
\begin{gathered}
\max _{x \in \mathbb{R}_{+}^{N}} \sum_{k i \in \mathcal{N}} v_{k i}\left(x_{k i}\right) \\
\text { s.t. } \quad \sum_{k \in \mathcal{K}^{l}} \max _{i \in \mathcal{G}_{k}^{l}}\left\{\alpha_{k i}^{l} x_{k i}\right\} \leqslant c^{l} \quad \forall l \in \mathcal{L}
\end{gathered}
$$


here $\mathcal{K}^{l}$ represents the set of multicast groups present on link $l$ and $\mathcal{G}_{k}^{l}$ represents the set of sub-indices from multicast group k, that are present on link l. Mathematically, the important property to note above is that the feasibility region is indeed a polytope (since the constraints are piecewise linear). We can easily linearize the above by using auxiliary variables $m_{k}^{l}$ as proxies for $\max _{i \in \mathcal{S}_{k}^{l}}\left\{\alpha_{k i}^{l} x_{k i}\right\}$ indexed by group and link as follows

$$
\begin{gathered}
\max _{x, m} \sum_{k i \in \mathcal{N}} v_{k i}\left(x_{k i}\right) \\
\text { s.t. } \quad \alpha_{k i}^{l} x_{k i} \leqslant m_{k}^{l} \quad \forall i \in \mathcal{G}_{k}^{l}, k \in \mathcal{K}^{l}, l \in \mathcal{L} \\
\\
\text { s.t. } \quad \sum_{k \in \mathcal{K}^{l}} m_{k}^{l} \leqslant c^{l} \quad \forall l \in \mathcal{L} .
\end{gathered}
$$

In the presence of auxiliary variables, the construction of the mechanism (esp. taxes) are slightly different in nature but follow the same philosophy as in this paper. Readers may refer to [Kakhbod and Teneketzis, 2013b], [Sinha and Anastasopoulos, 2013] for a full implementing mechanism specifically for the multi-rate multicast problem. Other works for the multicast problem include finding decentralized algorithms to achieve social utility maximizing allocation [Kar et al., 2001], [Stoenescu et al., 2007] as well as determining optimal allocation via max-min fairness [Sarkar and Tassiulas, 1999], [Sarkar and Tassiulas, 2002]. The incorporation of this model into the unified design methodology is a research topic the authors are currently working on.

The third possible generalization would be where the constraint set, instead of being a polytope, is taken as general convex set (satisfying assumptions from Section 2.3). If one represents the centralized problem in the form a general convex optimization problem

$$
\begin{array}{ll} 
& \max _{x, m} \sum_{i \in \mathcal{N}} v_{i}\left(x_{i}\right) \\
\text { s.t. } & g_{l}(x) \leqslant 0 \quad \forall l \in \mathcal{L},
\end{array}
$$

then, looking at the Lagrangian

$$
\mathrm{L}(x, \lambda)=\sum_{i \in \mathcal{N}} \nu_{i}\left(x_{i}\right)-\sum_{i \in \mathcal{L}} \lambda_{l} g_{l}(x),
$$

one can construct the first term (payment) in the tax (see (17b)) in a similar way as before. After this, the rest of tax terms can be analogously constructed from the KKT conditions.

The aforementioned generalizations together can lead to fully implementing mechanisms with minimal message space that can solve an even larger class of problems of interest. 
We conclude with a note on the robustness properties of these mechanisms that we would like to investigate. Recently there has been a lot of work related to robustness in mechanism design?. In addition to investigating the learning properties of our mechanism, we are also interested in investigating robustness of our mechanism (w.r.t. information i.e. beliefs of agents) and how the two might be related.

\section{References}

Sichao Yang and Bruce Hajek. Revenue and stability of a mechanism for efficient allocation of a divisible good. preprint, 2005.

Rajiv T Maheswaran and Tamer Basar. Social welfare of selfish agents: motivating efficiency for divisible resources. In Decision and Control, 2004. CDC. 43rd IEEE Conference on, volume 2, pages 1550-1555. IEEE, 2004.

A. Kakhbod and D. Teneketzis. Correction to "An efficient game form for unicast service provisioning". 2013a. URL http://arxiv.org/abs/0910.5502.

Ali Kakhbod and Demosthen Teneketzis. Correction to "An efficient game form for multirate multicast service provisioning". 31(7):1355-1356, 2013b. ISSN 0733-8716. doi: 10.1109/JSAC.2013.130717.

Abhinav Sinha and Achilleas Anastasopoulos. Generalized proportional allocation mechanism design for multi-rate multicast service on the internet. Technical report, 2013. URL http: //arxiv . org/abs/1307.2569.

Shrutivandana Sharma and Demosthenis Teneketzis. Local public good provisioning in networks: A Nash implementation mechanism. 30(11):2105-2116, 2012.

Zhu Han. Game theory in wireless and communication networks: theory, models, and applications. Cambridge University Press, 2012.

Yan Zhang and Mohsen Guizani. Game theory for wireless communications and networking. CRC Press, 2011.

Jianwei Huang, Randall A Berry, and Michael L Honig. Auction-based spectrum sharing. Mobile Networks and Applications, 11(3):405-418, 2006.

\footnotetext{
${ }^{7}$ see [Aghion et al., 2012] for treatment of the same and [Bergemann and Morris, 2013] for a survey of the major robustness results in Mechanism design.
} 
Michael J Neely. Optimal pricing in a free market wireless network. Wireless Networks, 15(7):901-915, 2009.

Tilman Börgers. An Introduction to the Theory of Mechanism Design. 2013. URL http://www-personal.umich.edu/ tborgers/TheoryOfMechanismDesign.pdf.

Dinesh Garg, Y Narahari, and Sujit Gujar. Foundations of mechanism design: A tutorial part 1-key concepts and classical results. In Sadhana (Academy Proceedings in Engineering Sciences), volume 33, pages 83-130. Indian Academy of Sciences, 2008a.

Matthew $O$ Jackson. A crash course in implementation theory. Social choice and welfare, 18(4):655-708, 2001.

Stefan Reichelstein and Stanley Reiter. Game forms with minimal message spaces. Econometrica: Journal of the Econometric Society, pages 661-692, 1988.

Theodore Groves and John Ledyard. Optimal allocation of public goods: A solution to the "free rider" problem. Econometrica: Journal of the Econometric Society, pages 783-809, 1977.

Jonh F. Nash. Non-cooperative Games. PhD thesis, Princeton University, May 1950.

Vijay Krishna. Auction theory. Academic press, 2009.

Yoav Shoham and Kevin Leyton-Brown. Multiagent systems: Algorithmic, gametheoretic, and logical foundations. Cambridge University Press, 2009.

Dinesh Garg, Y Narahari, and Sujit Gujar. Foundations of mechanism design: A tutorial part 2-advanced concepts and results. Sadhana, 33(2):131-174, 2008b.

Ramesh Johari and John N Tsitsiklis. Efficiency of scalar-parameterized mechanisms. Operations Research, 57(4):823-839, 2009.

Sichao Yang and Bruce Hajek. VCG-Kelly mechanisms for allocation of divisible goods: Adapting VCG mechanisms to one-dimensional signals. Selected Areas in Communications, IEEE Journal on, 25(6):1237-1243, 2007.

Frank P Kelly, Aman K Maulloo, and David KH Tan. Rate control for communication networks: shadow prices, proportional fairness and stability. Journal of the Operational Research society, 49(3):237-252, 1998.

Abhinav Sinha and Achilleas Anastasopoulos. Generalized proportional allocation mechanism design for unicast service on the internet. Technical report, 2014 . URL http://arxiv.org/abs/1401.1760. 
Rahul Jain and Jean Walrand. An efficient Nash-implementation mechanism for network resource allocation. Automatica, 46(8):1276-1283, 2010.

Leonid Hurwicz. Outcome functions yielding Walrasian and Lindahl allocations at Nash equilibrium points. The Review of Economic Studies, 46(2):217-225, 1979.

Yan Chen. A family of supermodular Nash mechanisms implementing Lindahl allocations. Economic Theory, 19(4):773-790, 2002.

Andreu Mas-Colell, Michael Dennis Whinston, Jerry R Green, et al. Microeconomic theory, volume 1. Oxford university press New York, 1995.

Paul J Healy and Laurent Mathevet. Designing stable mechanisms for economic environments. Theoretical Economics, 7(3):609-661, 2012.

H Peyton Young. Strategic learning and its limits. Oxford University Press, 2004.

Drew Fudenberg. The theory of learning in games, volume 2. MIT press, 1998.

Hamidou Tembine. Distributed strategic learning for wireless engineers. CRC Press, 2012.

Ishai Menache and Asuman Ozdaglar. Network games: Theory, models, and dynamics. Synthesis Lectures on Communication Networks, 4(1):1-159, 2011.

Samson Lasaulce and Hamidou Tembine. Game theory and learning for wireless networks: fundamentals and applications. Access Online via Elsevier, 2011.

Koushik Kar, Saswati Sarkar, and Leandros Tassiulas. Optimization based rate control for multirate multicast sessions. In INFOCOM 2001. Twentieth Annual Joint Conference of the IEEE Computer and Communications Societies. Proceedings. $I E E E$, volume 1, pages 123-132. IEEE, 2001.

Tudor Mihai Stoenescu, Mingyan Liu, and Demosthenis Teneketzis. Multirate multicast service provisioning $\mathrm{i}$ : an algorithm for optimal price splitting along multicast trees. Mathematical Methods of Operations Research, 65(2):199-228, 2007.

Saswati Sarkar and Leandros Tassiulas. Fair allocation of utilities in multirate multicast networks. volume 37, pages 718-727, 1999.

S. Sarkar and L. Tassiulas. Fair allocation of utilities in multirate multicast networks: a framework for unifying diverse fairness objectives. Automatic Control, IEEE Transactions on, 47(6):931-944, 2002. ISSN 0018-9286. doi: 10.1109/TAC.2002.1008359. 
Philippe Aghion, Drew Fudenberg, Richard Holden, Takashi Kunimoto, and Olivier Tercieux. Subgame-perfect implementation under information perturbations*. The Quarterly Journal of Economics, 127(4):1843-1881, 2012.

Dirk Bergemann and Stephen E Morris. An Introduction to Robust Mechanism Design. Now, 2013. 